PROCEEDINGS OF THE

AMERICAN MATHEMATICAL SOCIETY

Volume 138, Number 10, October 2010, Pages 3749-3752

S 0002-9939(10)10393-1

Article electronically published on May 6, 2010

\title{
AN EXAMPLE CONCERNING THE MENGER-URYSOHN FORMULA
}

\author{
JAN VAN MILL AND ROMAN POL
}

(Communicated by Alexander N. Dranishnikov)

\begin{abstract}
We construct subsets $A, B$ of the Euclidean space $\mathbb{R}^{4}$ such that $\operatorname{dim}(A \cup B)>\operatorname{dim}(A \times B)+1$. This provides a counterexample to a conjecture by E. Ščepin for subspaces of $\mathbb{R}^{4}$.
\end{abstract}

\section{INTRODUCTION}

In 1991, E. Ščepin conjectured ([D], the end of section 6, and [D-D], the beginning of section 5, $[\mathrm{L}-\mathrm{L}]$, page 74$)$ that the classical Menger-Urysohn formula $\operatorname{dim}(A \cup B) \leq$ $\operatorname{dim} A+\operatorname{dim} B+1$ can be improved by the formula $\operatorname{dim}(A \cup B) \leq \operatorname{dim}(A \times B)+1$. Since $\operatorname{dim}(A \times B) \leq \operatorname{dim} A+\operatorname{dim} B$, a positive answer to Ščepin's conjecture would indeed be an improvement. The question was repeated recently by V. Chatyrko $[$ ], Question 17. After this paper was completed, we were informed by M. Levin that from the results in $\mathrm{D}$ one can derive an example of a 5 -dimensional compactum $Z=A \cup B$ with $\operatorname{dim}(A \times B) \leq 3$.

Ščepin's conjecture is true in $\mathbb{R}^{6}$ if the union of $A$ and $B$ is $\sigma$-compact. Indeed, if $X=A \cup B$ is a $\sigma$-compact set in $\mathbb{R}^{6}$ and $\operatorname{dim} X \geq 5$ this follows from a deep theorem of A. Dranishnikov [D, Theorem B in section 6, as $\operatorname{dim}(X \times X)=2 \operatorname{dim} X$; cf. $[\mathrm{F}$, Ch. $5, \S 2$ and $\S 4$. Also, as was pointed out by M. Levin, if $\operatorname{dim} X \leq 4$, then the inequality $\operatorname{dim} X \leq \operatorname{dim}(A \cup B)+1$ can be derived from [D], Proposition 6.3 (even if the $\sigma$-compact set $X$ does not embed in $\mathbb{R}^{6}$ ).

The aim of this paper is to show that Ščepin's conjecture is not always true if the union of $A$ and $B$ is a $G_{\delta}$-subset of $\mathbb{R}^{4}$.

Example 1.1. There is a 3 -dimensional $G_{\delta}$-set $X$ in $\mathbb{R}^{4}$ which can be split into two sets, $X=A \cup B$, such that each finite product of the free union $A \oplus B$ is 1-dimensional. In particular,

$$
\operatorname{dim}(A \cup B)>\operatorname{dim}\left[(A \times B)^{m}\right]+1
$$

for all $m$. Moreover, $A$ is a $G_{\delta}$-set in $X$ (and hence, $B$ is an $F_{\sigma}$-subset of $X$ ).

To get the example, we put together some ideas and results from our earlier papers vM-P1 and vM-P2 concerning weakly $n$-dimensional sets; cf. section 2 .

Received by the editors August 7, 2009 and, in revised form, January 12, 2010.

2010 Mathematics Subject Classification. Primary 54F45, 55M10.

Key words and phrases. Menger-Urysohn formula, dimension, weakly $n$-dimensional, dimension of product.

(C)2010 American Mathematical Society Reverts to public domain 28 years from publication 


\section{Preliminaries}

We shall deal exclusively with subspaces of the Euclidean spaces $\mathbb{R}^{n}$. Our terminology follows $\left[\mathrm{K}\right.$. Given a space $X$, we denote by $X_{(n)}$ the set of points in $X$ that have arbitrarily small neighbourhoods with at most $(n-1)$-dimensional boundaries; cf. [K], $\mathrm{vM}$. We say that $X$ is weakly $n$-dimensional if $\operatorname{dim} X=n$ and $\operatorname{dim}\left(X \backslash X_{(n-1)}\right) \leq n-1, n \geq 1$.

We shall use the following result; cf. vM], Corollary 3.11 .12 and Exercise 2 for $\S 3.11$.

Theorem 2.1. The product of a countable family of weakly 1-dimensional spaces is 1-dimensional.

Let $\pi: \mathbb{R}^{n} \rightarrow \mathbb{R}$ be the projection onto the first coordinate and let $C \subset \mathbb{R}$ be the Cantor set. We shall need the following fact; cf. Lelek [L, Example, p. 80; Rubin, Schori and Walsh [R-S-W], Example 4.5; or [vM], proof of Theorem 3.9.3.

Proposition 2.2. For each $n$ there is a compact set $K_{n} \subset \mathbb{R}^{n+1}$ such that $\pi\left(K_{n}\right)=$ $C$ and each set $M \subset K_{n}$ with $\pi(M)=C$ is n-dimensional.

We end this section with a handy observation, used in [vM-P2] and vM], proof of Theorem 3.11.8.

Lemma 2.3. Let $f: E \rightarrow T$ be a perfect map from an $m$-dimensional space onto a zero-dimensional space. Then there exists a $G_{\delta}$-set $F$ in $E$ such that $f(F)=T$ and $\operatorname{dim}\left(F \backslash F_{(0)}\right) \leq m-1$.

Let us sketch a justification of this fact. We set $T_{0}=\left\{t \in T: \operatorname{dim} f^{-1}(t)=0\right\}$ and choose a zero-dimensional $F_{\sigma}$-set $M$ in $E$ such that $\operatorname{dim}(E \backslash M) \leq m-1$; cf. vM], Lemma 3.11.6. Then $F=f^{-1}\left(T_{0}\right) \cup(E \backslash M)$ has the required properties.

\section{Construction of Example 1.1}

We adopt the notation introduced in section 2 . Let us denote by $K$ the compact set $K_{3}$ described in Proposition 2.2, and let $p=\pi \mid K: K \rightarrow C$ be the restriction of the projection, mapping $K$ onto the Cantor set $C$.

The surjection $p$ has the following property:

(1) if $M \subset K$ and $p(M)=C$, then $\operatorname{dim} M=3$.

We let

(2) $S=\left\{t \in C: \operatorname{dim} p^{-1}(t)=0\right\}, \quad T=\left\{t \in C: 1 \leq \operatorname{dim} p^{-1}(t) \leq 2\right\}$.

Then (cf. vM], Lemma 3.11.7),

(3) $p^{-1}(S) \subset K_{(0)}$.

Let $\mathbb{P} \subset \mathbb{R}$ be the irrationals, and let

(4) $A=p^{-1}(S) \cup\left(\left(C \times \mathbb{P}^{3}\right) \cap K\right)$.

Then, by (3) and $[\mathrm{K}], 45, \mathrm{IV}$,

(5) $\operatorname{dim}\left(A \backslash A_{(0)}\right) \leq 0$; hence $\operatorname{dim} A \leq 1$, and $A$ is a $G_{\delta}$-set in $\mathbb{R}^{4}$.

Let us check that (cf. (2))

(6) $p(A) \supset C \backslash T$. 
Indeed, if $t \in C \backslash(S \cup T)$, then by $(2), p^{-1}(t)$ is a 3 -dimensional set in $\{t\} \times \mathbb{R}^{3}$. Hence it has nonempty interior in this section (cf. [vM], Theorem 3.7.1) and so there is $u \in \mathbb{P}^{3}$ such that $(t, u) \in p^{-1}(t) \cap K$, i.e., $t \in p(A)$; cf. (4).

Let us now consider the set $Y=p^{-1}(T)$. Then, by (2), $\operatorname{dim} Y \leq 2$ (cf. [vM], Lemma 3.6.10), and let us choose a zero-dimensional $G_{\delta^{-}}$set $G$ in $Y$ such that $\operatorname{dim}(Y \backslash G) \leq 1$. By (2), each fiber $p^{-1}(t)$ with $t \in T$ has positive dimension; hence $p^{-1}(t) \backslash G \neq \emptyset$. It follows that $f(Y \backslash G)=T$. Now, one can find countably many subsets of $Y \backslash G$ that are closed in $Y$ and whose images under $p$ are pairwise disjoint and cover $T$; cf. vM-P3, Lemma 2.1 (or vM-P1, section 4). In effect, we have sets $E_{i} \subset Y$ such that

(7) $E_{i}$ is closed in $p^{-1}(T), \quad \operatorname{dim} E_{i} \leq 1$,

(8) $p\left(E_{i}\right) \cap p\left(E_{j}\right)=\emptyset$ for $i \neq j, \bigcup_{i} p\left(E_{i}\right)=T$.

We use Lemma 2.3 to get sets $F_{i}$ such that

(9) $F_{i}$ is a $G_{\delta}$-set in $E_{i}, p\left(F_{i}\right)=p\left(E_{i}\right)$,

(10) $\operatorname{dim}\left(F_{i} \backslash\left(F_{i}\right)_{(0)}\right) \leq 0$.

Finally, we set (cf. (4))

(11) $B=\bigcup_{i} F_{i}$ and $X=A \cup B$.

Since the sets $p\left(E_{i}\right)$ are closed in $T$ (cf. (7)), from (8), (9) and the countable sum theorem, we infer that

(12) $F_{i}$ is closed in $B, \operatorname{dim} B \leq 1$, and $B$ is a $G_{\delta^{-}}$set in $p^{-1}(T)$.

By [K], $\S 45, \mathrm{IV}, p^{-1}(S \cup T)$ is a $G_{\delta^{-}}$set in $\mathbb{R}^{4}$; hence (4), (5) and (12) yield that (13) $X$ is a $G_{\delta^{-}}$set in $\mathbb{R}^{4}$.

By (8), (9) and (11), we also have $p(B)=T$, and hence $p(X)=C ;($ cf. $(6))$. Therefore, by (1),

(14) $\operatorname{dim} X=3$.

In particular, since by (5) and (12) the sets $A$ and $B$ are at most 1-dimensional, the Menger-Urysohn formula shows that they both have positive dimension. By (5),

(15) $A$ is weakly 1 -dimensional.

By (12) and the countable sum theorem, one of the sets $F_{i}$, say $F_{1}$, is 1-dimensional, and considering the sets $F_{1} \cup F_{i}$ we conclude from (10) that

(16) $B$ is covered by countably many closed weakly 1-dimensional sets.

It follows from (15) and (16) that any product $(A \oplus B)^{m}$ of the free union of $A$ and $B$ is covered by countably many closed sets, each of which is a product of weakly 1-dimensional spaces. By Theorem 2.1 and the countable sum theorem, $\operatorname{dim}\left((A \oplus B)^{m}\right)=1$.

\section{A comment}

We can repeat the construction described in section 3 for any $n \geq 3$, starting from the compact set $K_{n} \subset \mathbb{R}^{n+1}$ described in Proposition 2.2. Then we get as a result an $n$-dimensional $G_{\delta}$-set $X \subset \mathbb{R}^{n+1}$ and a decomposition $X=A \cup B$ such that $A$ is a weakly 1 -dimensional $G_{\delta}$-set in $\mathbb{R}^{n+1}$ and $B$ is a countable union of closed weakly $(n-2)$-dimensional sets. Using a theorem of Tomaszewski [T], vM-P2], it follows that $\operatorname{dim}(A \times B) \leq 1+(n-2)-1=n-2$. In effect, for the $n$-dimensional space $X$ we have $\operatorname{dim} X>\operatorname{dim}(A \times B)+1=n-1$. 


\section{REFERENCES}

[C] V. A. Chatyrko, Classical dimension theory, Open Problems in Topology. II (E. Pearl, ed.), North-Holland Publishing Co., Amsterdam, 2007, pp. 621-632.

[D] A. N. Dranishnikov, On the dimension of the product of two compacta and the dimension of their intersection in general position in Euclidean space, Trans. Amer. Math. Soc. 352 (2000), 5599-5618. MR1781276 (2001j:55002)

[D-D] A. N. Dranishnikov and J. Dydak, Extension theory of separable metrizable spaces with applications to dimension theory, Trans. Amer. Math. Soc. 353 (2001), 133-156. MR:1694287 (2001f:55002)

[F] V. V. Fedorčuk, The Fundamentals of Dimension Theory, Encyclopedia of Mathematical Sciences (A. V. Arhangel'skiı̌ and L. S. Pontrjagin, eds.), vol. 17, Springer-Verlag, Berlin, 1990, pp. 91-202.

[K] K. Kuratowski, Topology I, Academic Press, New York, 1966; Topology II, Academic Press, New York, 1968. MR0217751 (36:840) MR0259835 (41:4467)

[L] A. Lelek, Dimension inequalities for unions and mappings of separable metric spaces, Coll. Math. 23 (1971), 69-91. MR0322829 (48:1190)

[L-L] M. Levin, W. Lewis, Some mapping theorems for extensional dimension, Israel J. Math. 133 (2003), 61-76. MR.1968422 (2004d:54032)

[vM] J. van Mill, The infinite-dimensional topology of function spaces, North-Holland Publishing Co., Amsterdam, 2001. MR:1851014 (2002h:57031)

[vM-P1] J. van Mill and R. Pol, Note on weakly n-dimensional spaces, Monatsh. Math. 132 (2001), 25-33. MR 1825717(2002a:54027)

[vM-P2] J. van Mill and R. Pol, On spaces without non-trivial subcontinua and the dimension of their products, Topology Appl. 142 (2004), 31-48. MR2071291 (2005e:54034)

[vM-P3] J. van Mill and R. Pol, A complete $C$-space whose square is strongly infinitedimensional, Israel J. Math. 154 (2006), 209-220. MR.2254540 (2007h:54008)

[R-S-W] L. Rubin, R. M. Schori, and J. J. Walsh, New dimension-theory techniques for constructing infinite-dimensional examples, Gen. Top. Appl. 10 (1979), 93-102. MR519716 (80e:54049)

[T] B. Tomaszewski, On weakly n-dimensional spaces, Fund. Math. 103 (1979), 1-8. MR.535830 (80k:54067)

Department of Mathematics, Faculty of Sciences, VU University Amsterdam, De Boelelaan 1081, 1081 HV Amsterdam, The Netherlands

E-mail address: vanmill@few.vu.nl

Institute of Mathematics, University of Warsaw, Banacha 2, 02-097 Warszawa, POLAND

E-mail address: r.pol@mimuw.edu.pl 